\title{
Strategies of Luminescent Gold Nanoclusters for Chemo-/Bio-Sensing
}

\author{
Zhi He ${ }^{1,3}$, Tong Shu 1,3,*(D), Lei Su ${ }^{1,2,3, * \mathbb{D}}$ and Xueji Zhang 1,3,4,* \\ 1 Research Center for Biomedical and Health Science, Anhui Science and Technology University, \\ Fengyang 233100, China \\ 2 Beijing Advanced Innovation Center for Food Nutrition and Human Health, \\ Beijing Technology and Business University, Beijing 100048, China \\ 3 Research Center for Bioengineering and Sensing Technology, School of Chemistry and Bioengineering, \\ University of Science \& Technology Beijing, Beijing 100083, China \\ 4 School of Biomedical Engineering, Shenzhen University Health Science Center, Shenzhen 518060, \\ Guangdong, China \\ * Correspondence: shutong@ustb.edu.cn (T.S.); sulei@ustb.edu.cn (L.S.); zhangxueji@ustb.edu.cn (X.Z.)
}

Received: 15 July 2019; Accepted: 12 August 2019; Published: 22 August 2019

\begin{abstract}
Recent booming advances in luminescent gold nanoclusters (AuNCs), have prompted the development of novel fluorescent sensors. The luminescent AuNCs possess unique and intriguing physical and chemical properties including responsive photoluminescence and peroxide-like activity, providing abundant potentials for sensing strategy design. As of now, a wide variety of chem-/bio-sensors based on AuNCs have been developed and reviewed according to varied analytes. In this review, from a different point of view, we follow the route of how those sensors realize their functions and focus on the actual roles AuNCs play, in order to hierarchically and logically display the recent progress in the sensing applications of AuNCs. This review not only opens new windows to understand the development of sensors based on AuNCs but can also inspire broader and deeper utilization of luminescent nanomaterials.
\end{abstract}

Keywords: gold nanoclusters; photoluminescence; peroxide-like activity; chemo-/bio-sensing

\section{Introduction}

Gold nanoclusters (AuNC), composed of several atoms to several hundred unequal gold atoms, have attracted considerable interest because of their intriguing aesthetic structures and desired photophysical properties [1-14]. Their ultrasmall size $(<2 \mathrm{~nm})$, which is comparable to the Fermi wavelength of electrons, hampers the surface movement of valence electrons, giving rise to discrete electronic transitions and resulting interesting molecular properties, e.g., molecular chirality, HOMO-LUMO transitions and photoluminescence [15]. Compared with other metal nanoclusters, e.g., silver NCs [16] and copper NCs [17], AuNCs show superior stability against oxidation. Moreover, different from organic clusters, [18] luminescent AuNCs have also exhibited properties of long lifetime, large Stokes shift, and biocompatibility, facilitating to their practical applications in chemo-/bio-sensing [19,20].

Briefly, chemo-/bio-sensing is the process of converting analyte signals to readable electrical or optical signals. As for a luminescent sensor, the key of its construction lies proper luminescence materials, whose luminescent signals, e.g., intensity and wavelength, are specifically responsive to analytes [21]. As one of the attractive luminescence materials, AuNCs have experienced extensive growth in the past two decades. In the early stage, studies of AuNCs mainly proceeded in size-focusing structure analysis favored by the rapid development of organic chemistry [22]. Rare reports on their 
applications in sensing are there, mainly due to the lack of effective synthetic methods of AuNCs with desirable luminescence and aqueous solubility [23]. In 2009, Xie et al. reported a facile and "green" approach to synthesize AuNCs with strong fluorescence, excellent water solubility and biocompatibility [24]. The utility of commercial proteins, bovine serum albumin (BSA), as reducers and templates for AuNCs raised a wave of novel AuNCs stabilized by biomolecules, spurring the emergence of various analytical methods based on AuNCs. In 2012, they introduced the well-known concept of aggregation-induced emission (AIE) (proposed by Tang in 2001 [25-27]) into the scope of AuNC studies, igniting interest to study the responsive luminescence behaviors of AuNCs and their corresponding applications [28]. In short, the rapid development of synthetic approaches of luminescence AuNCs has also extensively pushed forward their applications in chemo-/bio-sensing [29-32].

In this review, we summarize recent sensing applications of AuNCs from the novel aspect of the roles they played in fulfilling conversion from analyte signals to luminescent signals. In each role, we describe in detail, the sensing strategies according to the responsiveness of AuNCs. In addition, we address intriguing peroxide properties of AuNCs and their use for colorimetric sensors. Finally, we outline current challenges and discuss future prospects of AuNCs for chem-/bio-sensing.

\section{Structure-Luminescence Relationship of AuNCs}

The luminescent properties of AuNCs are directly linked to the molecular structure of AuNCs. Core sizes are firstly unearthed and accepted to make crucial contributions to the luminescence of AuNCs. Size-dependent luminescence of AuNCs was suggested by a series of experimental studies [33]. The water-soluble dendrimers, polyamidoamine (PAMAM), with well-defined molecular weight was successfully applied to acquire a series of different sizes of $A u N C s$, including $A u_{5}, A u_{8}, A u_{13}, A u_{23}$, and $\mathrm{Au}_{31}$, which were determined by electrospray ionization mass spectrometry (ESI-MS) [34,35]. A distribution of luminescence spectra, varying from ultraviolet (UV) to near-infrared (NIR), were also correspondingly correlated to the sizes of AuNCs (Figure 1). The empirical formula on the size-dependent luminescence of the PAMAM-protected AuNCs was thus deduced: $E_{\text {Fermi }} / \mathrm{N}^{1 / 3}$ (where $\mathrm{E}_{\mathrm{Fermi}}$ is the Fermi energy of bulk gold, while $\mathrm{N}$ is the number of atoms). The energy scaling law is accurately suitable to the relationship for small AuNCs. As for larger AuNCs, a modified formula is required to correct the deviation from the electronic screening effects and the harmonic distortion in their potential energy well. Their corresponding modified free-electron models of the Au core (from spherical harmonic to Woods-Saxon and eventually to square-well potential), could be further used to explain the evolution of luminescence of AuNCs according to their increasing sizes [36].
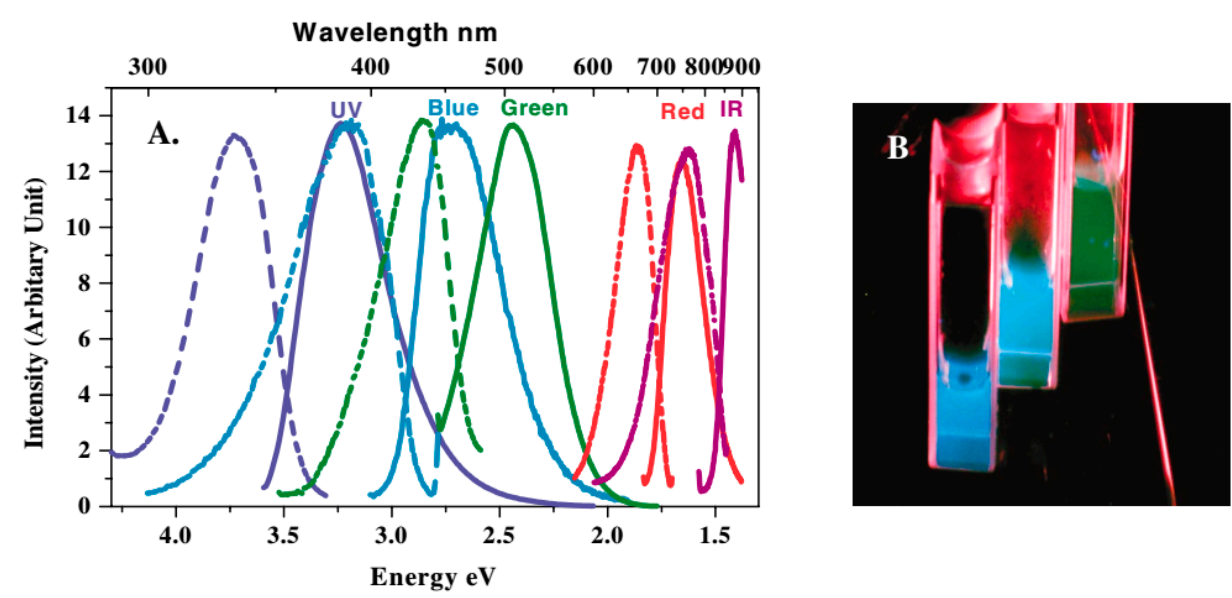

Figure 1. (A) Excitation (dashed) and emission (solid) spectra of polyamidoamine (PAMAM)-protected gold nanoclusters (AuNCs) with varied sizes. (B) Emission from the three shortest wavelength emitting AuNCs solutions (from left to right) under UV irradiation. Adapted with permission from Reference [34] Copyright (2004) American Physical Society Sites. 
Ligands also played a key role in the photoluminenscence of AuNCs. For example, the PL properties of the atomically precise glutathione (GSH)-protected AuNCs remain ambiguous with respect to their core size, which is in striking contrast with the dendrimer-protected AuNCs [23]. The type of thiolate ligands has been further studied and shown to affect the luminescence of thiolated $\mathrm{Au}_{25} \mathrm{NCs}$ [37]. It has thus been understood that electron-rich atoms or functional groups in the thiolate ligands can enhance the luminescence of the $\mathrm{Au}_{25} \mathrm{NCs}$. In addition, increasing the electro-positivity of the Au core could also promote the luminescent intensity of thiolated AuNCs.

\section{Synthetic Routes of AuNCs}

AuNCs were first reported by Naldini et al. in 1966 using phosphine as a ligand [38]. Since then, abundant physical and chemical methods have been developed to synthesize AuNCs of a size of $<2 \mathrm{~nm}$. Generally, similar to other nanomaterials, the synthetic routes of AuNCs can be divided into top-down and bottom-up routes.

\subsection{Top-Down Synthesis}

The etchants e.g., thiols and phosphines, have been recognized as the key tool to realize the top-down synthesis of AuNCs. These "scalpels" can segment large gold nanoparticles (AuNPs, core sizes $>2 \mathrm{~nm}$ ) into ultra-small AuNCs and then refine the relevant raw materials to size-focused AuNCs. For example, the core size of mercaptosuccinic acid (MSA)-protected AuNPs could be gradually reduced by adding excess BSA in alkaline conditions, [39] finally generating fluorescent BSA-protected AuNCs with the emission maximum appeared at $660 \mathrm{~nm}$. The particle size was unraveled to be about $1 \mathrm{~nm}$ with 38 gold atoms analyzed by MS. In the presence of GSH, the MSA-protected AuNPs could be well etched into two kinds of $\mathrm{AuNCs} \mathrm{Au}_{25}$, and $\mathrm{Au}_{8}$, respectively at $\mathrm{pH} 3$ and 7-8 [40]. Based on a similar top-down strategy, a variety of AuNCs could be synthesized successfully [41-43].

\subsection{Bottom-Up Synthesis}

The bottom-up synthesis undergoes a thoroughly controlled reduction of the $\mathrm{Au}^{3+}$ precursors under suitable ligand protection. $\mathrm{Au}^{3+}$ precursors and proper stabilizers are necessary for preparing luminescent AuNCs. Versatile AuNCs with different sizes and luminescence can be synthesized by controlling the reaction parameters, such as stabilizer, solvent, temperature, time, and $\mathrm{pH}$ of the solution. The stabilizers represented by proteins are the classic method to prepare luminescent AuNCs. Besides BSA, a series of proteins, such as urease, [44] catalase, [45] lysozyme, [46] plant proteins, [47] egg white, [48] goose feathers, [49] and globulin, [50] have been applied as stabilizers for the synthesis of luminescent AuNCs using a "one-pot" fashion method. The protein cages act as excellent scaffolds, which can regulate the growth of AuNCs within their well-defined ultrasmall 3D structures.

\section{Luminescent Sensing Strategies of AuNCs}

A typical sensing process consists of analyte identification, signal transition, and output [51]. Luminescent sensing strategies are based on sensors which can transfer analyte signals to readable photoluminescent signals. Of note, naked AuNCs have considerably high surface energy and hence a strong affinity to grow or aggregate into larger particles. The protections provided by ligands are necessary to maintain their solution stability [52]. Thus, we here denote metal cores and ligands together as AuNCs. In the sensing process, luminescent AuNCs can generate recordable photonic signals, which are closely related to their physicochemical structures. Therefore, sensing strategies targeting structures of AuNCs are the mainstream to develop various useful sensors. With these understandings, we then classify and describe recently-developed sensing strategies from multi-aspects, according to the roles of AuNCs within each sensor. 


\subsection{Mono-Component Sensing Systems Based on AuNCs}

Owing to the diversity of ligands and unique gold chemistry, AuNCs can be designed and developed into luminescent sensors. The richness of ligands provides abundant affinity sites interacted with targeted analytes, ensuring sensors with the desired selectivity. On the other hand, the Au core can interact with certain electron-donating groups (-S, $-\mathrm{CN},-\mathrm{P})$ and form strong Au-CN bonding in gold-cyanide complexes, Au-S bonding in gold-sulfur and Au-P bonding in gold-phosphine [53]. Additionally, the electronic configuration of the surface $A u^{+}$of AuNCs is $5 \mathrm{~d}^{10} 6 \mathrm{~s}^{0}$, a closed-shell configuration. Such configuration can exert highly specific and strong dispersion forces, which are greatly magnified by relativistic effects, to other closed-shell metal atoms, such as $\mathrm{Hg}^{2+}\left(5 \mathrm{~d}^{10} 6 \mathrm{~s}^{0}\right)$. The use of strong binding energies is therefore attractive for designing sensing approaches [54]. Hence, with proper modifications, versatile luminescent AuNCs are ready to sense analytes and give out detectable photons.

\subsubsection{Friendly Core-Targeting Strategies}

Analytes that only generate physical inhibition on the fluorescence of AuNCs, e.g., light absorption and electron transition, generally are not harmful towards AuNCs. AuNCs still maintain an intact structure and composition after interacting with analytes. Yang et al. found that phenol derivatives produced inner filter effects (IFE) on the emission of AuNCs [55]. They thus utilized blue-emitted AuNCs to quantitatively analyze toxic p-nitrophenol (pNP) and explosive trinitrotoluene (TNT). Particularly, the special closed-shell interactions of outer $\mathrm{Au}^{+}$with other ions with similar configuration can offer excellent selectivity and sensitivity for the corresponding ion detection. Xie et al. used BSA-protected AuNCs to construct a label-free sensor for $\mathrm{Hg}^{2+}$ and they pointed out that strong closed-shell interactions between $\mathrm{Au}^{+}$and $\mathrm{Hg}^{2+}$ were responsible for sensitively and selectively detecting $\mathrm{Hg}^{2+}$ (Figure 2) [54]. The unique interactions ensured that AuNCs place as powerful sensors for $\mathrm{Hg}^{2+}$, and inspired a series of relevant applications with novel AuNCs. Zang et al. reported new AuNCs using $\beta$-lactoglobulin as a reducer and stabilizer [56]. Using the protein-protected AuNCs, they realized the determination and quantification of $\mathrm{Hg}^{2+}$ in beverages, urine, and serum with high sensitivity and selectivity. On the other hand, the intactness of the AuNC core after interaction with $\mathrm{Hg}^{2+}$ hints that the removal of the cation can restore the origin emission of AuNCs. On the basis of this mechanism, sensors for biothiols which can form stable S-Hg bonds with $\mathrm{Hg}^{2+}$ have been developed. Park et al. applied the weakly emitting $\mathrm{Hg}^{2+}-\mathrm{AuNC}$ complexes to establish a turn-on responsive biosensor for biothiols (Figure 3) [57]. Biothiols, such as cysteine (Cys), glutathione (GSH), and homocysteine (Hcy), were able to block $\mathrm{Hg}^{2+}$-induced fluorescent quenching via interacting with thiophilic $\mathrm{Hg}^{2+}$ and, as a result, reactivated the intense fluorescence of AuNCs.
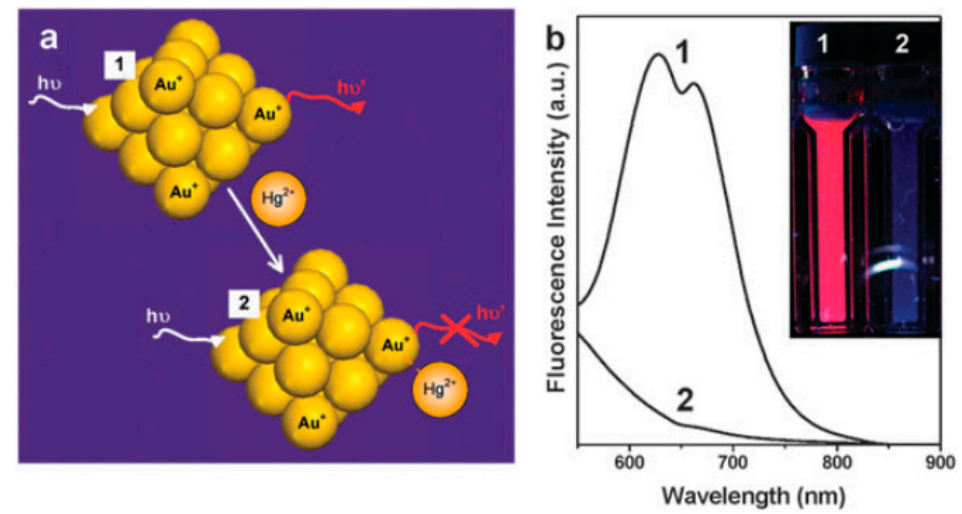

Figure 2. (a) Schematic of $\mathrm{Hg}^{2+}$ sensing based on the fluorescence quenching of AuNCs resulting from high-affinity metallophilic $\mathrm{Hg}^{2+}-\mathrm{Au}^{+}$bonds. (b) Photoemission spectra and (inset) photographs under UV light of AuNCs before and after adding trace $\mathrm{Hg}^{2+}$. Adapted with permission from Reference [54] Copyright (2010) Royal Society of Chemistry. 


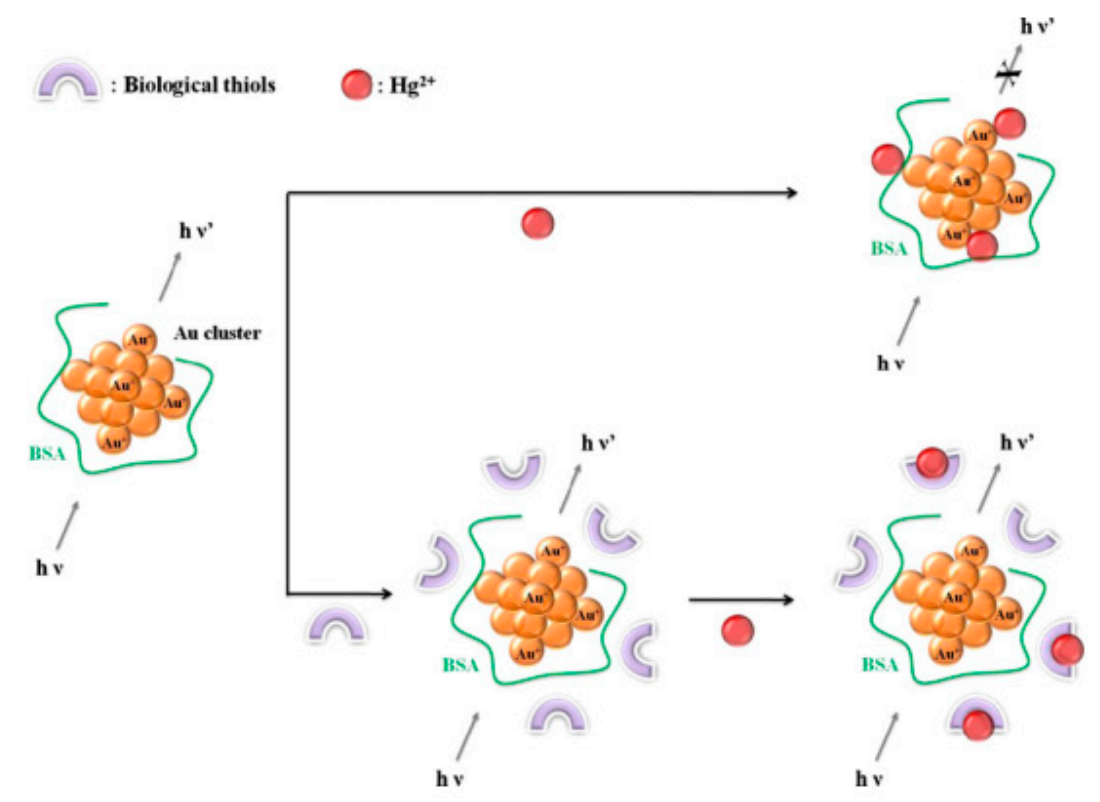

Figure 3. Schematic illustration of the biological thiol detection system based on the prevention against $\mathrm{Hg}^{2+}$-induced quenching of fluorescent gold nanoclusters (AuNCs). Adapted with permission from Reference [57] Copyright (2013) Elsevier.

\subsubsection{Core-Targeting Strategies with Gold Chemistry}

The chemical inertness of gold materials benefits them as sensors not only with good stability but also with selectivity over many potential interferences. In general, strong oxidants and specific coordinating agents can react with gold, finally resulting in quenching of AuNC fluorescence. Jiang's group found that the fluorescence of GSH-modified fluorescent could be significantly quenched by $\mathrm{hROS}\left(\bullet \mathrm{OH}, \mathrm{ClO}^{-}\right.$, and $\left.\mathrm{ONOO}^{-}\right)$instead of wROS $\left(\mathrm{H}_{2} \mathrm{O}_{2}\right.$ and $\left.\bullet \mathrm{O}^{2-}\right)$ at the same concentration, showing their excellent selectivity on hROS over wROS (Figure 4) [58]. They argued that the hROS-mediated fluorescence quenching was ascribable to the oxidation of AuNCs by hROS at both the Au sites and GSH sites. Similarly, Gopu et al. utilized the strong oxidizing agent, $\mathrm{HOCl}$, towards the gold core of BSA-AuNCs for fluorimetric detection of $\mathrm{HOCl}$ [59]. This rapid and simple method enabled sensitive determination of $\mathrm{HOCl}$ with a detection limit of $0.1 \times 10^{-6} \mathrm{M}$ with high selectivity over common ions present in real water samples.

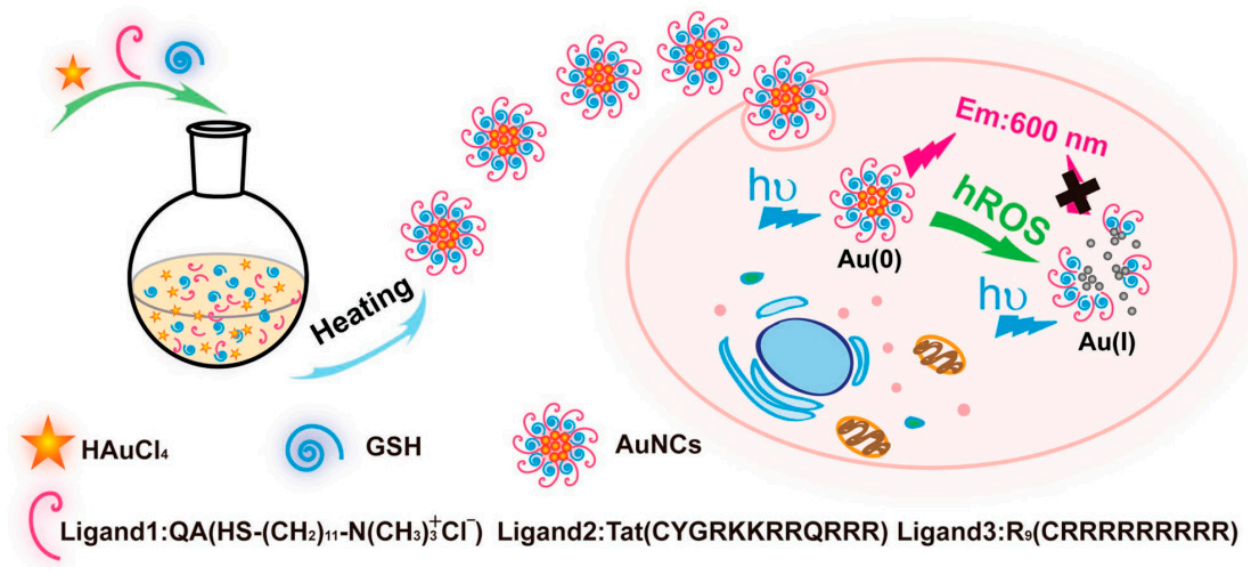

Figure 4. Schematic illustration of the one-step synthesis of red-emitting AuNCs with versatile surface chemistry, and the fluorescence quenching strategy for the living cell imaging of $\mathrm{hROS}\left(\bullet \mathrm{OH}, \mathrm{ClO}^{-}\right.$, and $\mathrm{ONOO}^{-}$) using the functionalized AuNCs. Adapted with permission from Reference [58] Copyright (2018) Wiley. 
In recent years, our groups have been dedicated to developing novel biosensors based on AuNC etching chemistry. The physio/pathologically important etchants were able to digest the gold core of fluorescence AuNCs into non-emissive $\mathrm{Au}^{+}$complexes. We first found that cysteamine (CSH), the main drug for pediatric nephropathic cystinosis, could selectively quench the fluorescence of BSA-protected AuNCs over Cys and GSH [60]. Simultaneously, the addition of CSH could bleach the brown-red color of AuNC solution into colorless. A deeper investigation unearthed that CSH triggered core-etching of AuNCs and oxidized elementary $\mathrm{Au}$ into $\mathrm{Au}(0)$ with strong supports from data of transmission electron microscopy (TEM), X-ray photoelectron spectroscopy (XPS), and matrix-assisted laser desorption/ionization time-of-flight mass spectrometry (MALDI-TOF-MS) [60]. Thus, we constructed a label-free and separation-free facile method for CSH detection and the method showed an excellent selectivity over common biothiols. Subsequently, we undertook massive works on etchant screening and successfully found that tris (2-carboxyethyl)phosphine (TCEP), a commonly-used chemical in reducing disulfide bonds, aroused a similar etching phenomenon towards AuNCs as CSH (Figure 5) [61]. The fact that TCEP created a chemical etching towards the AuNCs was then revealed by relevant characterizations. This method showed a linear relationship in the TCEP concentration range of $5 \times 10^{2}$ to $5 \times 10^{4} \mathrm{nM}$, achieving the highest sensitivity compared with previous methods. Following this etching strategy, we used a different kind of AuNCs, GSH-AuNCs, as the fluorescence probe. We found that GSH-AuNCs could be effectively etched by Cys and thus it could be applied to the facile, selective, and sensitive detection of cysteine. The sensor exhibited an ultra-wide linear concentration range as wide as nine orders of magnitude and an ultra-low limit of detection (LOD) of $6.3 \mathrm{pM}(\mathrm{S} / \mathrm{N}=3)[62]$.

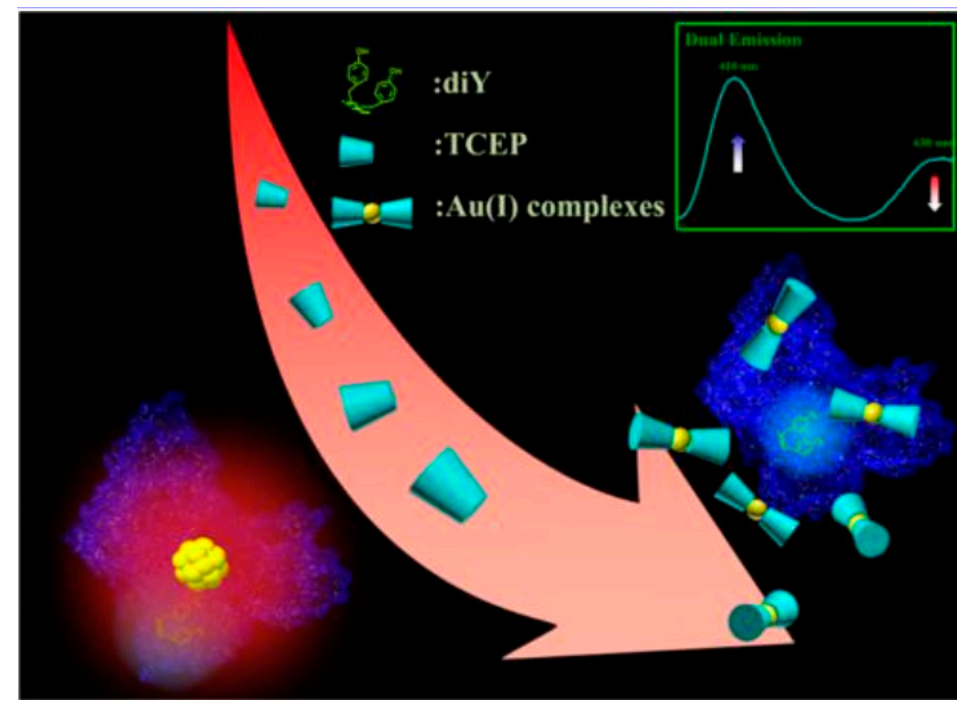

Figure 5. Tris (2-carboxyethyl)phosphine (TCEP) etching-induced quenching of red fluorescence emission of bovine serum albumin (BSA)-protected AuNCs and enhancing of the blue fluorescence emission of the dityrosine (diTyr) residues of the BSA ligands for detecting TCEP. Adapted with permission from Reference [61] Copyright (2016) American Chemistry Society.

\subsubsection{Ligands Targeting Strategies}

Ligands are of considerable importance to AuNCs. Ligand shells of AuNCs can be tailored independently, constituting versatile platforms for the development of simple and facile analytical methods. Wen et al. reported the synthesis of horseradish peroxidase-protected AuNCs through biomineralization, which were linearly responsive to $\mathrm{H}_{2} \mathrm{O}_{2}$ (Figure 6) [63]. They pointed out that the fluorescence quenching should be ascribed to $\mathrm{H}_{2} \mathrm{O}_{2}$-induced oxidation of thiolates in Au-S of AuNCs, leaving AuNCs unprotected and thus aggregated into large particles. Ligand exchanges of AuNCs can be alternatives for sensor design. Ligands of the acetylcysteine-stabilized AuNCs were reported to be 
able to be replaced by $\mathrm{S}^{2-}$ and the ligand exchange unstabilized AuNCs, causing the increase of the particle size and ultimately fluorescence quenching [64]. The "turn-off" phenomenon thus could be used to selectively detect $\mathrm{H}_{2} \mathrm{~S}$. Polymethylacrylic acid (PMMA) was reported to be able to incorporate AuNCs [65]. Their abundant carboxyl groups were capable of coupling the high-valence cation, $\mathrm{Fe}^{3+}$, rendering aggregation of AuNCs and hence fluorescence quenching. A sensitive fluorescence biosensor for $\mathrm{Fe}^{3+}$ was thus developed using PMMA-protected AuNCs. Specifically, enzyme-templated AuNCs show a significant affinity to their substrates. West et al. synthesized AuNCs DNase 1 as templates [66]. They found that the DNase 1 after AuNC synthesis still retained the endodeoxyribonuclease activity. Thus, DNA was able to bind with the luminescent hybrids and quench the emission of AuNCs.
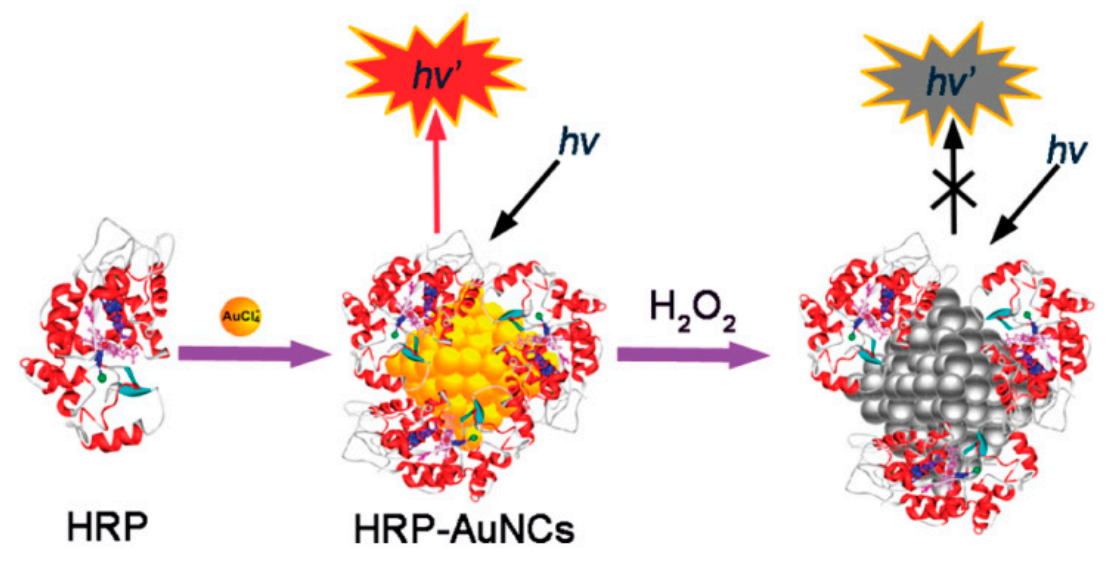

Figure 6. Schematic of the formation and the $\mathrm{H}_{2} \mathrm{O}_{2}$ directed quenching of horseradish peroxidase (HRP)-protected AuNCs. Adapted with permission from Reference [63] Copyright (2011) American Chemistry Society.

The aggregated AuNCs by crosslinking their ligands are responsive materials for developing powerful sensors. You et al. reported the use of the aggregated AuNCs prepared by Ce(III)-induced assembly of glutathione-capped AuNCs for alkaline phosphatase (ALP) detection [67]. The introduction of Ce(III) significantly enhanced the emission of AIE-type AuNCs due to cation-induced aggregation. ATP was able to compete with GSH-AuNCs for Ce(III) binding, resulting in partially quenching of the assembly. The competitive quenching could be significantly inhibited by ALP, which could catalytically degrade ATP and thus recover enhanced luminescence of AuNCs. Dai et al. crosslinked BSA-templated AuNCs with disulfide bonds and prepared self-quenched aggregated AuNCs. The disulfide bonds could be reductively cleaved by GSH, which restored the fluorescence of quenched AuNCs. The GSH-triggered fluorescence recovery was further used to develop GSH sensors with desirable sensitivity and selectivity. Furthermore, ligand modification with a specific recognition group can equip AuNCs with a responsivity towards more species. Ban et al. functionalized AuNCs with $\mathrm{NH}_{2}-\beta$-cyclodextrin (CD) to develop sensors for dopamine (Figure 7) [68]. The grafted CD on AuNCs could selectively bind dopamine by non-covalent interaction of supramolecular host-guest recognition, which promoted the energy transfer from AuNCs to dopamine and hence quenched the fluorescence of AuNCs.

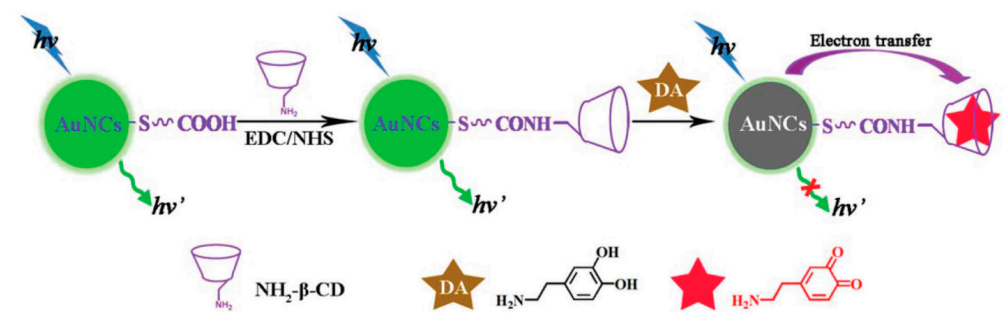

Figure 7. Schematic illustration of the analytical strategy for dopamine detection. Adapted with permission from Reference [68] Copyright (2015) Royal Chemistry Society. 


\subsection{Dual-Components Sensing Systems Based on AuNCs and X}

Although solo AuNCs can adequately act as sensors, their suitable analytes are limited. A further extension of sensor components can immensely enrich and strengthen this system. The $\mathrm{X}$ materials could introduce new interactions and physico-chemical properties into the sensing system, which can be used to design and develop novel sensing sensors.

\subsubsection{Fluorescence-Quenched System}

The partner materials in this system generally are electron-/energy-receiver, which can efficiently quench the fluorescence of AuNCs. Separation or removal of the quencher from AuNCs can restore the fluorescence of AuNCs. Liu et al. found that p-nitrophenyl phosphate (PNPP) was capable of effectively quenching mercaptoundecanoic acid (MUA)-protected AuNCs through IFE [69]. They thus utilized the quenching system to detect ALP, which could degrade PNPP and eliminate IFE, resulting in the recovery of AuNC fluorescence. Carbon nanomaterials are known as benign and broad quenchers. Liu et al. used nanosized graphene oxide (GO) to efficiently quench the emission of MUA-protected AuNCs through the Forster resonance energy transfer (FRET) pathway [70]. The interaction between GO and AuNCs was bridged by positively-charged peptides. The charge of the bridge could be reversed by modifying phosphate groups, which were introduced by protein kinase (PKA), resulting in the attenuation of the interaction of GO with peptides and destruction of FRET between MUA-AuNCs and GO. The following fluorescence recovery of AuNCs could be applied to quantitatively detect PKA. Large gold nanomaterials are another good electron-/energy-receiver. Liu et al. presented a universal and facile one-step strategy for sensitive and selective detection of pathogenic bacteria using a dual-molecular affinity-based FRET platform based on the recognition of bacterial cell walls by antibiotic and aptamer molecules, respectively [71]. They employed vancomycin (Van) and a nucleic acid aptamer to functionalize AuNCs as the energy donor and AuNPs as the energy acceptor, respectively. Staphylococcus aureus could thus selectively bridge the two components and activate the FRET process. Qin et al. constructed a quenching assembly with GSH-protected AuNCs and amine-terminated gold nanorods (AuNRs) [72]. Glutathione S-transferase (GSST) could competitively bind GSH-protected AuNCs and restore their fluorescence, enabling a linear detection of GSST. $\mathrm{MnO}_{2}$ 2D nanosheets recently received much attention for their potential as a responsive fluorescence quencher. Yan et al. wrapped AuNCs with $\mathrm{MnO}_{2}$ nanosheets, forming nanostructured hybrids (Figure 8) [73]. The fluorescence of AuNCs was quenched by the nanosheets through the FRET effect. The effect could be obviated by oxidatively decomposing $\mathrm{MnO}_{2}$ nanosheets by $\mathrm{H}_{2} \mathrm{O}_{2}$, [73] resulting in the luminescence recovery of AuNCs. $\mathrm{H}_{2} \mathrm{O}_{2}$ could be further enzymatically produced by oxidizing cetylcholine, thus enabling the system to detect cetylcholine and its derivatives.
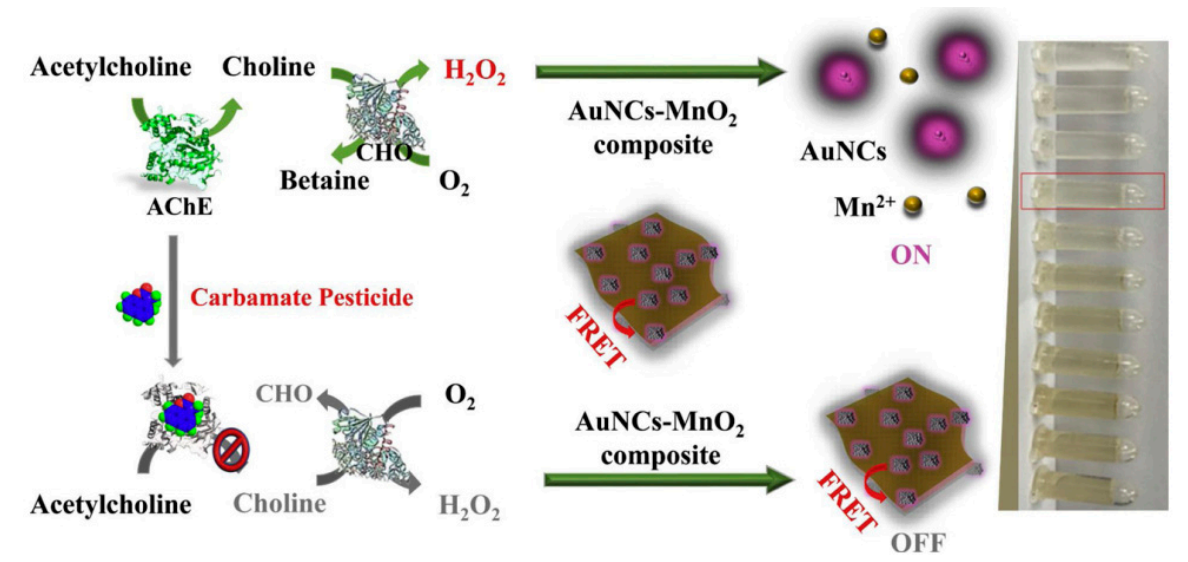

Figure 8. Schematic illustration of the dual-signal system for detecting cetylcholine and its derivatives. Adapted with permission from Reference [41] Copyright (2019) Elsevier. 


\subsubsection{Fluorescence-Enhanced System}

Recently, the type of AuNCs with surprising AIE characteristics has attracted growing interest and been widely applied to develop novel sensors. The fluorescence of AIE-type AuNCs is considerably enhanced in aggregated states. Thus, the elimination of the factors that can induce the aggregation of AuNCs can be an efficient way to design sensors. Lin et al. reported a novel nanoassembly of amino-functionalized mesoporous silica nanoparticle(MSN)-AuNCs (Figure 9) [74]. The assembling force remarkably enhanced the emission of AuNCs, which, however, could be inhibited in the presence of negatively charged heparin due to the stronger interaction between heparin and MSNs. The de-enhancement could be used to sensitively detect heparin. Similarly, Xue et al. found that the amino-modified silicon nanoparticles (SiNPs) could significantly enhance GSH-capped AuNCs through electrostatic interaction [75]. The novel nanohybrids possessed a dual-emission property and could be used as a ratiometric probe. Cationic protamine exhibited stronger binding affinity towards AuNCs and could compete with SiNPs, resulting in inhibition of the self-assembly and hence the fluorescence quenching of GSH-AuNCs. The competition from protamine could be largely suppressed by trypsin, which could catalyze the hydrolysis of protamine. Thus, the dual-emissive system could be used to quantitatively determinate trypsin. The general phenomenon that the emission of GSH-protected AuNCs could be significantly enhanced via multi-positively-charged species, inspired You et al. to develop a sensing platform for various analytes [76]. They found that poly-arginine could trigger the AIE enhancement of GSH-protected AuNCs, indicating the feasibility of peptides to enhance the emission of AuNCs. Further designs of four biosensors were proposed using different positively-charged peptides, which could specifically interact with heparin, trypsin, ALP, and integrin. The four analytes thus could remove peptide and quench the enhanced luminescence of AuNCs.

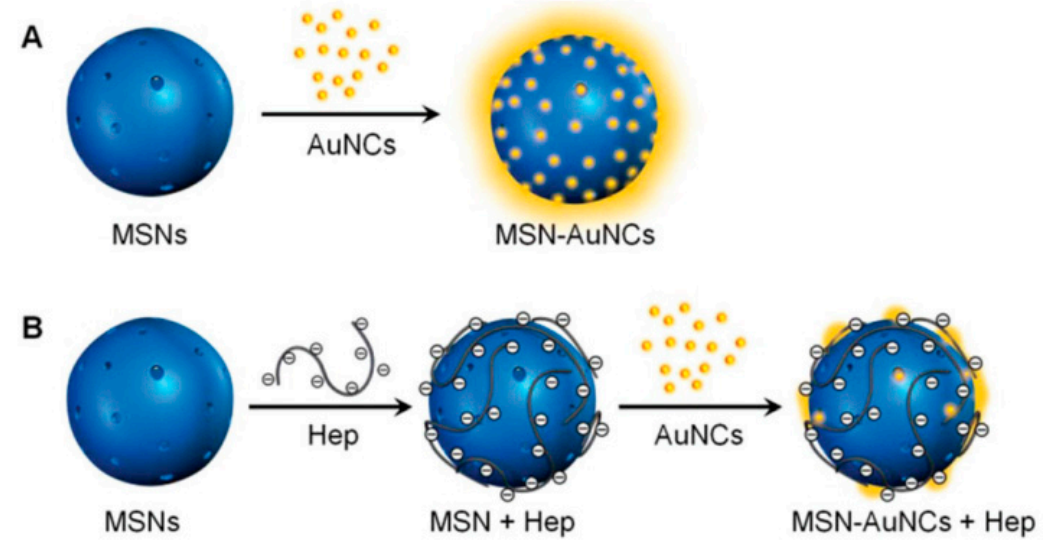

Figure 9. Schematics illustrations of (A) the self-assembly of mesoporous silica nanoparticle (MSN)-AuNC nanocomposites, and (B) the detection of heparin. Adapted with permission from Reference [46] Copyright (2018) Royal Chemistry Society.

\subsubsection{Ratiometric Sensing System}

The fluorometric process is easily affected by variations from longtime-used illumination sources, environmental noises, and sample position, resulting in an instability of acquired data. The introduction of an extra fluorophore as a reference can satisfactorily overcome those drawbacks. Hence, ratiometric sensors consisted of AuNCs as a probe or reference have aroused increasing attention. Our group has revealed the synthesis of strong blue-emitted dityrosine (diTyr) during AuNC formation within BSA [77]. After thoroughly investigating the luminescence properties of the duel emissive system, we developed a ratiometric sensor for $\mathrm{p}$-nitrophenol (pNP), which was able to effectively quench the emission of diTyr by IFE rather than that of AuNCs (Figure 10) [78]. Further, Deng et al. developed this response for enzyme analysis [44]. They used the selective quenching of diTyr emissions by pNP to design an analytical ratiometric approach for sulfatase, which could catalyze p-nitrophenyl 
sulfate and then release pNP. Wang's group recently reported a sensitive and selective ratiometric fluorescence sensing platform to detect tyrosinase (TYR) activity and dopamine using GSH-protected AuNCs as probes [79]. The emission of AuNCs could be effectively quenched by quinones, while TYR could catalyze the oxidization of dopamine to o-quinone, therefore quenching the fluorescence of AuNCs. Meanwhile, the reaction of TYR and dopamine generated an emissive complex located at $400 \mathrm{~nm}$, facilitating the construction of a ratiometric biosensor for TYR activity and dopamine. Recently, carbon dots (CDs), as fluorescence nanomaterials, are known for their ease to synthesize and excellent biocompatibility. Wang et al. reported a novel ratiometric fluorescent probe for $\mathrm{Pb}^{2+}$ composed by positively-charged CDs and negatively-charged AuNCs [80]. $\mathrm{Pb}^{2+}$ could enhance the emission of AuNCs, while the CDs remained unchanged. Additionally, Yan et al. constructed a highly sensitive selective ratio fluorescence sensor for $\mathrm{Hg}^{2+}$ using a dual emission system of CDs and AuNCs [81]. $\mathrm{Hg}^{2+}$ could form close-shell interaction with surface $\mathrm{Au}^{+}$of AuNCs and considerably quench the red fluorescence of AuNCs, while the blue fluorescence of CDs is not affected by $\mathrm{Hg}^{2+}$. Modification of ligands with external organic luminophores are plausible approaches to construct ratiometric probes. $\mathrm{Yu}$ et al. developed a novel FRET-based ratiometric sensor using AuNCs as an energy donor and $\mathrm{H}_{2} \mathrm{~S}$-sensitive cyclamate derivative (Cy1) as energy receptors [82]. With this functionalized AuNCs, they could accurately detect $\mathrm{H}_{2} \mathrm{~S}$ in vitro and in vivo.

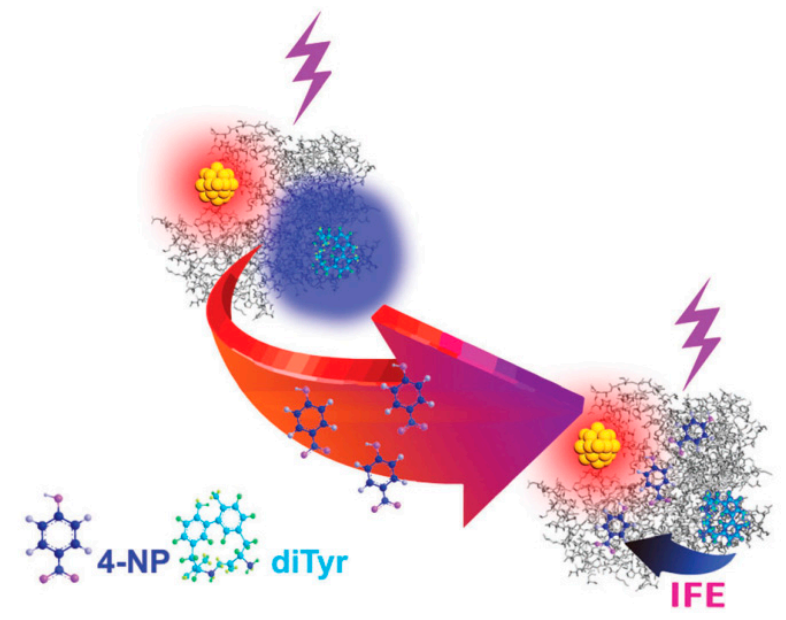

Figure 10. Schematic illustration of the 4-NP-induced selective quenching of the blue fluorescence emission of the diTyr residues of the GNCs@BSA via the inner filter effects (IFE). Adapted with permission from Reference [50] Copyright (2018) Royal Chemistry Society.

\subsection{Multi-Components Sensing Systems Based on Single AuNCs}

Sensing systems containing several elements are expected to be capable of undergoing more complicated tasks. For example, $\mathrm{Xu}$ et al. reported the construction of a core-shell composite nanostructure $\mathrm{Ag} @ \mathrm{SiO}_{2}$-AuNCs (composed of an outer conjugated layer of silver core, silica shell, and AuNCs) [83]. The interaction between AuNCs and the silver core enhances the fluorescence emission of AuNCs. A multi-component detection sensing system with the OFF-ON-OFF switching nature for $\mathrm{Cu}^{2+}$, inorganic pyrophosphate (PPi), and pyrophosphatase (PPase) could be developed. However, too many components in the system might introduce excess interferences and signal loss, resulting in making the sensing process cumbersome.

\section{Sensing Strategies Based on Peroxide Properties}

Interestingly, in the past few years, peroxide-like characteristics of AuNCs have been unearthed and have attracted increasing attention. Compared with natural enzymes, the AuNCs are more robust and can undergo a wide range of $\mathrm{pH}$ and temperature. Furthermore, among nanomaterial-based artificial enzymes, the AuNCs exhibit superior advantages for bioanalysis due to their easy modification, 
ultra-small size, and excellent biocompatibility. Liu et al. established a new type of colorimetric nanosensor for $\mathrm{Cu}^{2+}$ and histidine (His) using the intrinsic peroxidase properties of AuNCs (Figure 11) [84]. $\mathrm{Cu}^{2+}$ could inhibit the peroxidase activity of histidine-protected AuNCs nanoclusters and the inhibition could be offset by free His. Thus, highly sensitive and selective colorimetric nanosensors for $\mathrm{Cu}^{2+}$ and His could be constructed, due to the strong affinity between $\mathrm{Cu}^{2+}$ and His. $\mathrm{Hg}^{2+}$ can also inhibit the intrinsic peroxidase properties of AuNCs. By the removal of $\mathrm{Hg}^{2+}$ away from AuNCs with melamine (MA), Cao et al. constructed a colorimetric sensor for the quantitative detection of MA, which could restore $\mathrm{Hg}^{2+}$-induced inhibition of the enzymatic activity of AuNCs [85]. Feng et al. reported that the peroxidase-like activity of AuNCs could be suppressed by GSH binding [86]. They applied this phenomenon to develop a colorimetric assay for GSH and realized the identification of cancer cells, which generated much more GSH than normal cells.
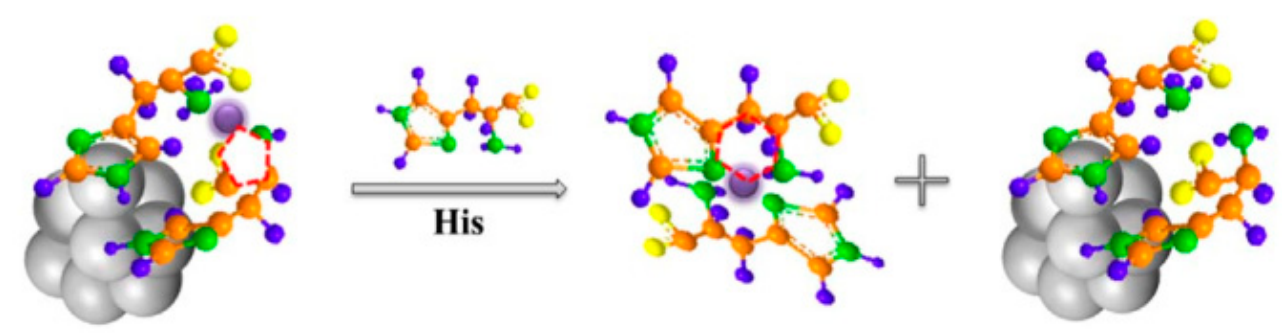

Figure 11. Schematic illustration of the coordination interaction of histidine (His)-AuNC with $\mathrm{Cu}^{2+}$, and His with $\mathrm{Cu}^{2+}$. Adapted with permission from Reference [84] Copyright (2017) Elsevier.

\section{Summary and Outlook}

As of now, a number of AuNCs with luminescence spanning from UV to near-IR has been reported. Compared with quantum dots (QDs), AuNCs are more biocompatible and can be readily bioconjugated. However, their quantum yield is normally much lower than that of QDs and organic dyes, greatly limiting signal harvesting. Therefore, although many efforts have been undertaken to improve their quantum yield, there is still a need for AuNCs with enhanced fluorescence in an aqueous solution.

Ligand shells play an essential role in stabilizing AuNCs and equipping the encapsulated AuNCs with extra functions. Ligand shells of AuNCs have been decorated with many organic materials, rendering sensing platforms based on AuNCs more versatile. Therefore, exploring new organic ligands for the preparation of AuNCs would further stimulate the design of new fluorescent sensing systems.

Currently, the majority of sensing strategies using AuNCs are one-to-one signal transfer (from analytes to photons) models. Technologies, such as enzymatic cycling amplification and nanotechnology amplification, are desired to further enhance the sensitivity of sensors based on AuNCs.

Similar to other nanomaterials, it is always a challenging thing to obtain AuNCs with uniform composition and structure. The uniformity endows the corresponding sensors or other applications with sound reproducibility. Although plausible progress has been achieved in the field of sized-focused AuNCs in organic solvents, considerable efforts are needed to expand such synthesis into aqueous systems.

In summary, the current strategies of AuNCs for sensing applications were systematically classified and discussed in detail according to their functions and roles. This review paves the way for others to logically utilize the unique properties of AuNCs and provides inspiration for their future applications in chem-/bio-sensing.

Author Contributions: Conceptualization, T.S.; investigation, T.S. and Z.H.; resources, Z.H.; writing-original draft preparation, Z.H. and T.S.; writing —review and editing, T.S. and L.S.; supervision, X.Z.; project administration, X.Z.; funding acquisition, T.S, L.S., and X.Z.

Funding: We are grateful for the financial support from, and greatly acknowledge the Beijing Natural Science Foundation (2184107), the Fundamental Research Funds for the Central Universities (FRF-BR-18-009B), Beijing Advanced Innovation Center for Food Nutrition and Human Health, Beijing Technology and Business University (20181035), and the National Natural Science Foundation of China (21890742). 
Conflicts of Interest: The authors declare no conflict of interest.

\section{References}

1. Yao, Q.; Feng, Y.; Fung, V.; Yu, Y.; Jiang, D.E.; Yang, J.; Xie, J. Precise control of alloying sites of bimetallic nanoclusters via surface motif exchange reaction. Nat. Commun. 2017, 8, 1555. [CrossRef] [PubMed]

2. Cordon, J.; Jimenez-Oses, G.; Lopez-de-Luzuriaga, J.M.; Monge, M. The key role of Au-substrate interactions in catalytic gold subnanoclusters. Nat. Commun. 2017, 8, 1657. [CrossRef] [PubMed]

3. Zhao, J.B.; Jin, R.C. Heterogeneous catalysis by gold and gold-based bimetal nanoclusters. Nano Today 2018, 18, 86-102. [CrossRef]

4. Wang, S.; Abroshan, H.; Liu, C.; Luo, T.Y.; Zhu, M.; Kim, H.J.; Rosi, N.L.; Jin, R. Shuttling single metal atom into and out of a metal nanoparticle. Nat. Commun. 2017, 8, 848. [CrossRef]

5. Zeng, C.; Chen, Y.; Kirschbaum, K.; Lambright, K.J.; Jin, R. Emergence of hierarchical structural complexities in nanoparticles and their assembly. Science 2016, 354, 1580-1584. [CrossRef] [PubMed]

6. Yu, M.X.; Zhou, C.; Liu, L.; Zhang, S.R.; Sun, S.S.; Hankins, J.D.; Sun, X.K.; Zheng, J. Interactions of Renal-Clearable Gold Nanoparticles with Tumor Microenvironments: Vasculature and Acidity Effects. Angew. Chem. Int. Edit. 2017, 56, 4314-4319. [CrossRef] [PubMed]

7. Xu, J.; Peng, C.Q.; Yu, M.X.; Zheng, J. Renal clearable noble metal nanoparticles: Photoluminescence, elimination, and biomedical applications. WIRES Nanomed. Nanobiotechnol. 2017, 9, e1453. [CrossRef] [PubMed]

8. Xie, Y.Z.Y.; Liu, Y.; Yang, J.C.; Liu, Y.; Hu, F.P.; Zhu, K.; Jiang, X.Y. Gold Nanoclusters for Targeting Methicillin-Resistant Staphylococcusaureus InVivo. Angew. Chem. Int. Ed. 2018, 57, 3958-3962. [CrossRef] [PubMed]

9. Huang, R.W.; Wei, Y.S.; Dong, X.Y.; Wu, X.H.; Du, C.X.; Zang, S.Q.; Mak, T.C.W. Hypersensitive dual-function luminescence switching of a silver-chalcogenolate cluster-based metal-organic framework. Nat. Chem. 2017, 9, 689-697. [CrossRef]

10. Santiago-Gonzalez, B.; Monguzzi, A.; Capitani, C.; Prato, M.; Santambrogio, C.; Meinardi, F.; Brovelli, S. Bottom-up Synthesis and Self-Assembly of Copper Clusters into Permanent Excimer Supramolecular Nanostructures. Angew. Chem. Int. Ed. 2018, 57, 7051-7055. [CrossRef] [PubMed]

11. Lei, Z.; Li, J.J.; Wan, X.K.; Zhang, W.H.; Wang, Q.M. Isolation and Total Structure Determination of an All-Alkynyl-Protected Gold Nanocluster Au144. Angew. Chem. Int. Ed. 2018, 57, 8639-8643. [CrossRef] [PubMed]

12. Santiago-Gonzalez, B.; Monguzzi, A.; Caputo, M.; Villa, C.; Prato, M.; Santambrogio, C.; Torrente, Y.; Meinardi, F.; Brovelli, S. Metal Nanoclusters with Synergistically Engineered Optical and Buffering Activity of Intracellular Reactive Oxygen Species by Compositional and Supramolecular Design. Sci. Rep. 2017, 7, 5976. [CrossRef] [PubMed]

13. Santiago-Gonzalez, B.; Monguzzi, A.; Azpiroz, J.M.; Prato, M.; Erratico, S.; Campione, M.; Lorenzi, R.; Pedrini, J.; Santambrogio, C.; Torrente, Y.; et al. Permanent excimer superstructures by supramolecular networking of metal quantum clusters. Science 2016, 353, 571-575. [CrossRef]

14. Zhang, H.; Liu, H.; Tian, Z.Q.; Lu, D.; Yu, Y.; Cestellos-Blanco, S.; Sakimoto, K.K.; Yang, P.D. Bacteria photosensitized by intracellular gold nanoclusters for solar fuel production. Nat. Nanotechnol. 2018, 13, 900-905. [CrossRef] [PubMed]

15. Chakraborty, I.; Pradeep, T. Atomically Precise Clusters of Noble Metals: Emerging Link between Atoms and Nanoparticles. Chem. Rev. 2017, 117, 8208-8271. [CrossRef] [PubMed]

16. Shu, T.; Lin, X.; Zhou, Z.; Zhao, D.; Xue, F.; Zeng, F.; Wang, J.; Wang, C.; Su, L.; Zhang, X. Understanding stimuli-responsive oligomer shell of silver nanoclusters with aggregation-induced emission via chemical etching and their use as sensors. Sens. Actuators B-Chem. 2019, 286, 198-205. [CrossRef]

17. Liu, X.; Astruc, D. Atomically precise copper nanoclusters and their applications. Coord. Chem. Rev. 2018, 359, 112-126. [CrossRef]

18. He, C.; Xiao, Y.; Huang, J.; Lin, T.; Mya, K.Y.; Zhang, X. Highly efficient luminescent organic clusters with quantum dot-like properties. J. Am. Chem. Soc. 2004, 126, 7792-7793. [CrossRef] 
19. Zhang, Y.; Zhang, C.; Xu, C.; Wang, X.; Liu, C.; Waterhouse, G.I.N.; Wang, Y.; Yin, H. Ultrasmall Au nanoclusters for biomedical and biosensing applications: A mini-review. Talanta 2019, 200, 432-442. [CrossRef]

20. Chen, L.-Y.; Wang, C.-W.; Yuan, Z.; Chang, H.-T. Fluorescent Gold Nanoclusters: Recent Advances in Sensing and Imaging. Anal. Chem. 2015, 87, 216-229. [CrossRef] [PubMed]

21. Jeong, Y.; Kook, Y.M.; Lee, K.; Koh, W.G. Metal enhanced fluorescence (MEF) for biosensors: General approaches and a review of recent developments. Biosens. Bioelectron. 2018, 111, 102-116. [CrossRef] [PubMed]

22. Huang, C.-C.; Yang, Z.; Lee, K.-H.; Chang, H.-T. Synthesis of Highly Fluorescent Gold Nanoparticles for Sensing Mercury(II). Angew. Chem. Int. Ed. 2007, 119, 6948-6952. [CrossRef]

23. Negishi, Y.; Nobusada, K.; Tsukuda, T. Glutathione-protected gold clusters revisited: Bridging the gap between gold(I)-thiolate complexes and thiolate-protected gold nanocrystals. J. Am. Chem. Soc. 2005, 127, 5261-5270. [CrossRef] [PubMed]

24. Xie, J.; Zheng, Y.; Ying, J.Y. Protein-directed synthesis of highly fluorescent gold nanoclusters. J. Am. Chem. Soc. 2009, 131, 888-889. [CrossRef]

25. Mei, J.; Leung, N.L.; Kwok, R.T.; Lam, J.W.; Tang, B.Z. Aggregation-Induced Emission: Together We Shine, United We Soar! Chem. Rev. 2015, 115, 11718-11940. [CrossRef] [PubMed]

26. Gao, M.; Tang, B.Z. Fluorescent Sensors Based on Aggregation-Induced Emission: Recent Advances and Perspectives. ACS Sens. 2017, 2, 1382-1399. [CrossRef] [PubMed]

27. Luo, J.; Xie, Z.; Lam, J.W.Y.; Cheng, L.; Chen, H.; Qiu, C.; Kwok, H.S.; Zhan, X.; Liu, Y.; Zhu, D.; et al. Aggregation-induced emission of 1-methyl-1,2,3,4,5-pentaphenylsilole. Chem. Commun. 2001, 18, 1740-1741. [CrossRef]

28. Luo, Z.; Yuan, X.; Yu, Y.; Zhang, Q.; Leong, D.T.; Lee, J.Y.; Xie, J. From aggregation-induced emission of $\mathrm{Au}(\mathrm{I})$-thiolate complexes to ultrabright $\mathrm{Au}(0) @ \mathrm{Au}(\mathrm{I})$-thiolate core-shell nanoclusters. J. Am. Chem. Soc. 2012, 134, 16662-16670. [CrossRef] [PubMed]

29. Su, Y.; Xue, T.; Liu, Y.; Qi, J.; Jin, R.; Lin, Z. Luminescent metal nanoclusters for biomedical applications. Nano Res. 2019, 6, 1251-1265.

30. Wang, B.; Zhao, M.; Mehdi, M.; Wang, G.; Gao, P.; Zhang, K.-Q. Biomolecule-assisted synthesis and functionality of metal nanoclusters for biological sensing: A review. Mater. Chem. Front. 2019. [CrossRef]

31. Dou, X.; Chen, X.; Zhu, H.; Liu, Y.; Chen, D.; Yuan, X.; Yao, Q.; Xie, J. Water-soluble metal nanoclusters: Recent advances in molecular-level exploration and biomedical applications. Dalton Trans. 2019. [CrossRef]

32. Khandelwal, P.; Poddar, P. Fluorescent metal quantum clusters: An updated overview of the synthesis, properties, and biological applications. J. Mater. Chem. B 2017, 5, 9055-9084.

33. Goswami, N.; Yao, Q.; Luo, Z.; Li, J.; Chen, T.; Xie, J. Luminescent Metal Nanoclusters with Aggregation-Induced Emission. J. Phys. Chem. Lett. 2016, 7, 962-975. [CrossRef]

34. Zheng, J.; Zhang, C.; Dickson, R.M. Highly Fluorescent, Water-Soluble, Size-Tunable Gold Quantum Dots. Phys. Rev. Lett. 2004, 93, 077402. [CrossRef] [PubMed]

35. Zheng, J.; Petty, J.T.; Dickson, R.M. High quantum yield blue emission from water-soluble Au8 nanodots. J. Am. Chem. Soc. 2003, 125, 7780-7781. [CrossRef]

36. Zheng, J.; Nicovich, P.R.; Dickson, R.M. Highly fluorescent noble-metal quantum dots. Annu. Rev. Phys. Chem. 2007, 58, 409-431. [CrossRef]

37. Wu, Z.; Jin, R. On the ligand's role in the fluorescence of gold nanoclusters. Nano Lett. 2010, 10, $2568-2573$. [CrossRef]

38. Naldini, L.; Cariati, F.; Simonetta, G.; Malatesta, L. Gold-tertiary phosphine derivatives with intermetallic bonds. Chem. Commun. 1966, 18, 647-648. [CrossRef]

39. Habeeb Muhammed, M.A.; Verma, P.K.; Pal, S.K.; Retnakumari, A.; Koyakutty, M.; Nair, S.; Pradeep, T. Luminescent quantum clusters of gold in bulk by albumin-induced core etching of nanoparticles: Metal ion sensing, metal-enhanced luminescence, and biolabeling. Chemistry 2010, 16, 10103-10112. [CrossRef] [PubMed]

40. Habeeb Muhammed, M.A.; Ramesh, S.; Sinha, S.S.; Pal, S.K.; Pradeep, T. Two distinct fluorescent quantum clusters of gold starting from metallic nanoparticles by $\mathrm{pH}$-dependent ligand etching. Nano. Res. 2010, 1, 333-340. [CrossRef] 
41. Zhu, M.; Lanni, E.; Garg, N.; Bier, M.E.; Jin, R. Kinetically controlled, high-yield synthesis of Au25 clusters. J. Am. Chem. Soc. 2008, 130, 1138-1139. [CrossRef] [PubMed]

42. Duan, H.; Nie, S. Etching colloidal gold nanocrystals with hyperbranched and multivalent polymers: A new route to fluorescent and water-soluble atomic clusters. J. Am. Chem. Soc. 2007, 129, 2412-2413. [CrossRef]

43. Qian, H.; Zhu, M.; Lanni, E.; Zhu, Y.; Bier, M.E.; Jin, R. Conversion of Polydisperse Au Nanoparticles into Monodisperse Au25 Nanorods and Nanospheres. J. Phys. Chem. C 2009, 113, 17599-17603. [CrossRef]

44. Deng, H.H.; Peng, H.P.; Huang, K.; He, S.B.; Yuan, Q.F.; Lin, Z.; Chen, R.; Xia, X.H.; Chen, W. Self-Referenced Ratiometric Detection of Sulfatase Activity with Dual-Emissive Urease-Encapsulated Gold Nanoclusters. ACS Sens. 2019, 4, 344-352. [CrossRef] [PubMed]

45. Meng, F.; Yin, H.; Li, Y.; Zheng, S.; Gan, F.; Ye, G. One-step synthesis of enzyme-stabilized gold nanoclusters for fluorescent ratiometric detection of hydrogen peroxide, glucose and uric acid. Microchem. J. 2018, 141, 431-437. [CrossRef]

46. Alkudaisi, N.; Russell, B.A.; Jachimska, B.; Birch, D.J.S.; Chen, Y. Detecting lysozyme unfolding via the fluorescence of lysozyme encapsulated gold nanoclusters. J. Mater. Chem. B 2019, 7, 1167-1175. [CrossRef]

47. Li, Z.; Peng, H.; Liu, J.; Tian, Y.; Yang, W.; Yao, J.; Shao, Z.; Chen, X. Plant Protein-Directed Synthesis of Luminescent Gold Nanocluster Hybrids for Tumor Imaging. ACS Appl. Mater. Interfaces 2018, 10, 83-90. [CrossRef] [PubMed]

48. Zhang, Y.; Jiang, J.J.; Li, M.; Gao, P.F.; Shi, L.H.; Zhang, G.M.; Dong, C.; Shuang, S.M. Bright far-red/near-infrared gold nanoclusters for highly selective and ultra-sensitive detection of $\mathrm{Hg}^{2+}$. Sens. Actuators B-Chem. 2017, 238, 683-692. [CrossRef]

49. Shu, T.; Cheng, X.; Wang, J.; Lin, X.; Zhou, Z.; Su, L.; Zhang, X. Synthesis of Luminescent Gold Nanoclusters Embedded Goose Feathers for Facile Preparation of $\mathrm{Au}(\mathrm{I})$ Complexes with Aggregation-Induced Emission. ACS Sustain. Chem. Eng. 2018, 7, 592-598. [CrossRef]

50. Xu, S.H.; Feng, X.Y.; Gao, T.; Wang, R.Z.; Mao, Y.N.; Lin, J.H.; Yu, X.J.; Luo, X.L. A novel dual-functional biosensor for fluorometric detection of inorganic pyrophosphate and pyrophosphatase activity based on globulin stabilized gold nanoclusters. Anal. Chim. Acta 2017, 958, 22-29. [CrossRef]

51. Mehrotra, P. Biosensors and their applications-A review. J. Oral Biol. Craniofac. Res. 2016, 6, 153-159. [CrossRef] [PubMed]

52. Shu, T.; Wang, J.X.; Li, X.Q.; Zhang, X.J.; Su, L. Fluorescent Film Sensors Based on Fluorescent Gold and Silver Nanoclusters. Curr. Nanosci. 2015, 11, 702-709. [CrossRef]

53. Britvin, S.N.; Lotnyk, A. Water-Soluble Phosphine Capable of Dissolving Elemental Gold: The Missing Link between 1,3,5-Triaza-7-phosphaadamantane (PTA) and Verkade's Ephemeral Ligand. J. Am. Chem. Soc. 2015, 137, 5526-5535. [CrossRef] [PubMed]

54. Xie, J.; Zheng, Y.; Ying, J.Y. Highly selective and ultrasensitive detection of $\mathrm{Hg}^{2+}$ based on fluorescence quenching of $\mathrm{Au}$ nanoclusters by $\mathrm{Hg}^{2+-} \mathrm{Au}^{+}$interactions. Chem. Commun. 2010, 46, 961-963. [CrossRef] [PubMed]

55. Yang, X.; Wang, J.; Su, D.; Xia, Q.; Chai, F.; Wang, C.; Qu, F. Fluorescent detection of TNT and 4-nitrophenol by BSA Au nanoclusters. Dalton Trans. 2014, 43, 10057-10063. [CrossRef] [PubMed]

56. Zang, J.; Li, C.; Zhou, K.; Dong, H.; Chen, B.; Wang, F.; Zhao, G. Nanomolar $\mathrm{Hg}^{2+}$ Detection Using beta-Lactoglobulin-Stabilized Fluorescent Gold Nanoclusters in Beverage and Biological Media. Anal. Chem. 2016, 88, 10275-10283. [CrossRef] [PubMed]

57. Park, K.S.; Kim, M.I.; Woo, M.A.; Park, H.G. A label-free method for detecting biological thiols based on

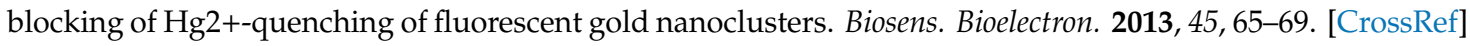
[PubMed]

58. Xie, Y.; Xianyu, Y.; Wang, N.; Yan, Z.; Liu, Y.; Zhu, K.; Hatzakis, N.S.; Jiang, X. Functionalized Gold Nanoclusters Identify Highly Reactive Oxygen Species in Living Organisms. Adv. Funct. Mater. 2018, 14, 1702026. [CrossRef]

59. Gopu, C.L.; Shanti Krishna, A.; Sreenivasan, K. Fluorimetric detection of hypochlorite using albumin stabilized gold nanoclusters. Sens. Actuators B-Chem. 2015, 209, 798-802. [CrossRef]

60. Shu, T.; Su, L.; Wang, J.; Li, C.; Zhang, X. Chemical etching of bovine serum albumin-protected Au25 nanoclusters for label-free and separation-free detection of cysteamine. Biosens. Bioelectron. 2015, 66, $155-161$. [CrossRef] 
61. Shu, T.; Wang, J.; Su, L.; Zhang, X. Chemical Etching of Bovine Serum Albumin-Protected Au25 Nanoclusters for Label-Free and Separation-Free Ratiometric Fluorescent Detection of Tris(2-carboxyethyl)phosphine. Anal. Chem. 2016, 88, 11193-11198. [CrossRef]

62. Wang, J.; Lin, X.; Su, L.; Yin, J.; Shu, T.; Zhang, X. Chemical etching of pH-sensitive aggregation-induced emission-active gold nanoclusters for ultra-sensitive detection of cysteine. Nanoscale 2018, 11, 294-300. [CrossRef]

63. Wen, F.; Dong, Y.; Feng, L.; Wang, S.; Zhang, S.; Zhang, X. Horseradish peroxidase functionalized fluorescent gold nanoclusters for hydrogen peroxide sensing. Anal. Chem. 2011, 83, 1193-1196. [CrossRef]

64. Zhang, Y.; Li, M.; Niu, Q.Q.; Gao, P.F.; Zhang, G.M.; Dong, C.; Shuang, S.M. Gold nanoclusters as fluorescent sensors for selective and sensitive hydrogen sulfide detection. Talanta 2017, 171, 143-151. [CrossRef]

65. Zheng, S.Y.; Yin, H.Q.; Li, Y.; Bi, F.L.; Gan, F. One-step synthesis of L-tryptophan-stabilized dual-emission fluorescent gold nanoclusters and its application for Fe3+ sensing. Sens. Actuators B-Chem. 2017, 242, 469-475. [CrossRef]

66. West, A.L.; Griep, M.H.; Cole, D.P.; Karna, S.P. DNase 1 retains endodeoxyribonuclease activity following gold nanocluster synthesis. Anal. Chem. 2014, 86, 7377-7382. [CrossRef] [PubMed]

67. You, J.G.; Lu, C.Y.; Krishna Kumar, A.S.; Tseng, W.L. Cerium(iii)-directed assembly of glutathione-capped gold nanoclusters for sensing and imaging of alkaline phosphatase-mediated hydrolysis of adenosine triphosphate. Nanoscale 2018, 10, 17691-17698. [CrossRef] [PubMed]

68. Ban, R.; Abdel-Halim, E.S.; Zhang, J.; Zhu, J.-J. beta-Cyclodextrin functionalised gold nanoclusters as luminescence probes for the ultrasensitive detection of dopamine. Analyst 2015, 140, 1046-1053. [CrossRef] [PubMed]

69. Liu, H.J.; Li, M.; Xia, Y.N.; Ren, X.Q. A Turn-On Fluorescent Sensor for Selective and Sensitive Detection of Alkaline Phosphatase Activity with Gold Nanoclusters Based on Inner Filter Effect. ACS Appl. Mater. Interfaces 2017, 9, 120-126. [CrossRef] [PubMed]

70. Liu, Q.; Li, N.; Wang, M.; Wang, L.; Su, X. A label-free fluorescent biosensor for the detection of protein kinase activity based on gold nanoclusters/graphene oxide hybrid materials. Anal. Chim. Acta 2018, 1013, 71-78. [CrossRef]

71. Yu, M.Q.; Wang, H.; Fu, F.; Li, L.Y.; Li, J.; Li, G.; Song, Y.; Swihart, M.T.; Song, E.Q. Dual-Recognition Forster Resonance Energy Transfer Based Platform for One-Step Sensitive Detection of Pathogenic Bacteria Using Fluorescent Vancomycin-Gold Nanoclusters and Aptamer-Gold Nanoparticles. Anal. Chem. 2017, 89, 4085-4090. [CrossRef]

72. Qin, L.; He, X.; Chen, L.; Zhang, Y. Turn-on Fluorescent Sensing of Glutathione S-Transferase at near-Infrared Region Based on FRET between Gold Nanoclusters and Gold Nanorods. ACS Appl. Mater. Interfaces 2015, 7, 5965-5971. [CrossRef] [PubMed]

73. Yan, X.; Kong, D.S.; Jin, R.; Zhao, X.; Li, H.X.; Liu, F.M.; Lin, Y.H.; Lu, G.Y. Fluorometric and colorimetric analysis of carbamate pesticide via enzyme-triggered decomposition of Gold nanoclusters-anchored $\mathrm{MnO} 2$ nanocomposite. Sens. Actuators B-Chem. 2019, 290, 640-647. [CrossRef]

74. Ma, L.; Zhang, M.; Yang, A.; Wang, Q.; Qu, F.; Qu, F.; Kong, R.-M. Sensitive fluorescence detection of heparin based on self-assembly of mesoporous silica nanoparticle-gold nanoclusters with emission enhancement characteristics. Analyst 2018, 143, 5388-5394. [CrossRef] [PubMed]

75. Xue, F.; Qu, F.; Han, W.; Xia, L.; You, J. Aggregation-induced emission enhancement of gold nanoclusters triggered by silicon nanoparticles for ratiometric detection of protamine and trypsin. Anal. Chim. Acta 2019, 1046, 170-178. [CrossRef] [PubMed]

76. You, J.-G.; Tseng, W.-L. Peptide-induced aggregation of glutathione-capped gold nanoclusters: A new strategy for designing aggregation-induced enhanced emission probes. Anal. Chim. Acta 2019, 1078, 101-111. [CrossRef] [PubMed]

77. Su, L.; Shu, T.; Wang, J.; Zhang, Z.; Zhang, X. Hidden Dityrosine Residues in Protein-Protected Gold Nanoclusters. J. Phys. Chem. C 2015, 119, 12065-12070. [CrossRef]

78. Shu, T.; Wang, J.; Lin, X.; Zhou, Z.; Liang, F.; Su, L.; Zhang, X. Dual-emissive gold nanoclusters for separation-free and label-free ratiometric fluorescent sensing of 4-nitrophenol based on inner filter effect. J. Mater. Chem. C 2018, 6, 5033-5038. [CrossRef]

79. Teng, Y.; Jia, X.; Li, J.; Wang, E. Ratiometric Fluorescence Detection of Tyrosinase Activity and Dopamine Using Thiolate-Protected Gold Nanoclusters. Anal. Chem. 2015, 87, 4897-4902. [CrossRef] 
80. Wang, L.; Cao, H.X.; He, Y.S.; Pan, C.G.; Sun, T.K.; Zhang, X.Y.; Wang, C.Y.; Liang, G.X. Facile preparation of amino-carbon dots/gold nanoclusters FRET ratiometric fluorescent probe for sensing of $\mathrm{Pb}^{2+} / \mathrm{C}+$. Sens. Actuators B-Chem. 2019, 282, 78-84. [CrossRef]

81. Yan, Y.H.; Yu, H.; Zhang, K.; Sun, M.T.; Zhang, Y.J.; Wang, X.K.; Wang, S.H. Dual-emissive nanohybrid of carbon dots and gold nanoclusters for sensitive determination of mercuric ions. Nano Res. 2016, 9, 2088-2096. [CrossRef]

82. Yu, Q.; Gao, P.; Zhang, K.Y.; Tong, X.; Yang, H.; Liu, S.; Du, J.; Zhao, Q.; Huang, W. Luminescent gold nanocluster-based sensing platform for accurate $\mathrm{H} 2 \mathrm{~S}$ detection in vitro and in vivo with improved anti-interference. Light-Sci. Appl. 2017, 6, e17107. [CrossRef] [PubMed]

83. Xu, D.D.; Zheng, B.; Song, C.Y.; Lin, Y.; Pang, D.W.; Tang, H.W. Metal-enhanced fluorescence of gold nanoclusters as a sensing platform for multi-component detection. Sens. Actuators B-Chem. 2019, 282, 650-658. [CrossRef]

84. Liu, Y.; Ding, D.; Zhen, Y.L.; Guo, R. Amino acid-mediated 'turn-off/turn-on' nanozyme activity of gold nanoclusters for sensitive and selective detection of copper ions and histidine. Biosens. Bioelectron. 2017, 92, 140-146. [CrossRef] [PubMed]

85. Cao, G.-X.; Wu, X.-M.; Dong, Y.-M.; Li, Z.-J.; Wang, G.-L. Colorimetric determination of melamine based on the reversal of the mercury(II) induced inhibition of the light-triggered oxidase-like activity of gold nanoclusters. Microchim. Acta 2016, 183, 441-448. [CrossRef]

86. Feng, J.Y.; Huang, P.C.; Shi, S.Z.; Deng, K.Y.; Wu, F.Y. Colorimetric detection of glutathione in cells based on peroxidase-like activity of gold nanoclusters: A promising powerful tool for identifying cancer cells. Anal. Chim. Acta 2017, 967, 64-69. [CrossRef] [PubMed]

(C) 2019 by the authors. Licensee MDPI, Basel, Switzerland. This article is an open access article distributed under the terms and conditions of the Creative Commons Attribution (CC BY) license (http://creativecommons.org/licenses/by/4.0/). 